\title{
Fetal scalp stimulation tests to improve the value of an admission cardiotocograph
}

\author{
Kumarapperuma K.A.U.S ${ }^{a}$, Goonewardene I.M.R ${ }^{b}$
}

\begin{abstract}
Introduction: An admission cardiotocograph (CTG) in a woman in early labour can lead to unnecessary interventions without improving neonatal outcomes
\end{abstract}

Objective: To assess fetal scalp stimulation as a complementary test to improve the value of an admission CTG.

Method: Women $(\mathrm{n}=243)$ with uncomplicated singleton pregnancies with a cephalic presentation at $>37$ weeks gestation, in early labour or with induction of labour (IOL )) had a CTG for ten minutes followed by a fetal scalp Digital Stimulation Test (DST) for 15 seconds during the routine vaginal examination, and the CTG was continued for another five minutes. If fetal heart rate (FHR) acceleration were absent after DST ( DST negative), an Allis Clamp was applied to the fetal scalp and the CTG was continued for another five minutes. FHR accelerations indicated a positive test result. The initial CTG and the results of DST and application of Allis Clamp were compared with the five-minute APGAR score of the neonate.

Results: Of the 243 women, 107 (44\%) had a negative DST but this number decreased to $27(11 \%)$ after additional Allis clamping of the fetal scalp. The addition of the fetal scalp stimulation tests (FSST) markedly increased the specificity of the CTG in predicting neonatal hypoxia from $58 \%$ (95\% CI 51.3-64.6\%) to $96.4 \%$ (95\% CI 93-98.5\%). After the addition of FSST, the likelihood ratio of the presence of FHR accelerations in excluding neonatal hypoxia was 22.1 (95\% CI 10.8-45.4) while the likelihood ratio of the absence of FHR accelerations in predicting neonatal hypoxia was $0.22(95 \%$ CI $0.1-0.52)$

Conclusion: When FHR accelerations are absent in the admission CTG, FSST complement it by helping to rule out fetuses probably not having hypoxia during early labour or IOL, and enables the identification of fetuses who would require close monitoring.

\section{INTRODUCTION}

In low risk women who are admitted in labour, a routine admission cardiotocograph (CTG) is not recommended as it leads to unnecessary interventions without improving neonatal outcomes. This is because a CTG per se is

acting Consultant Obstetrician and

Gynaecologist, Base Hospital,Udugama

${ }^{b}$ Professor of Obstetrics and Gynaecology, Department of Obstetrics and Gynaecology, Faculty of Medicine, Galle

Correspondence: Surange Kumarapperuma

Email-udayasurange@gmail.com

Competing interests: The authors report no conflict of interest not a good method of fetal monitoring as it has poor specificity with significant false positive rates ${ }^{[1]}$. Therefore the addition of a complementary test such as fetal scalp blood sampling is used in well-resourced settings ${ }^{[2]}$. However fetal scalp blood sampling is not feasible in many centers. Fetal stimulation tests such as the Fetal Acoustic Stimulation Test (FAST), fetal scalp Digital Stimulation Test (DST), application of an Allis Clamp to the scalp and fetal scalp puncture have also been shown to improve the value of the CTG by improving its specificity ${ }^{[3-6]}$. The FAST has been used effectively in the Academic Obstetrics and Gynaecology unit of the Teaching Hospital Mahamodara, Galle
(THMG) for both antenatal and early intra partum fetal monitoring for several years $[7,8]$.

The Digital Stimulation Test (DST) is easy to carry out during the vaginal examination by gently stroking the fetal scalp for 15 seconds ${ }^{[5,6]}$. It is non invasive, and no sophisticated instruments are required. Allis clamping is non traumatic pinching of the scalp with the Allis clamp for 15 seconds. Vigorous stroking and /or Allis clamping for longer durations should be avoided as it could lead to fetal vagal stimulation leading to bradycardia. With both these tests the non hypoxic fetus usually reacts to the stimulation with fetal heart rate (FHR) accelerations. Although the absence of accelerations per se does not indicate possible underlying fetal hypoxia, the occurrence of FHR accelerations would indicated that fetal hypoxia is unlikely although it is not possible to completely exclude it. Furthermore the occurrence of FHR accelerations would also enable shortening the duration of the CTG on the other hand, the absence of FHR accelerations following feta scalp stimulation tests

(FSST) would suggest the need for close monitoring of the fetus ${ }^{[3-6]}$. A metaanalysis of intra partum fetal stimulation tests have shown that, as the likely hood ratios of negative tests are very low, these tests are useful in ruling out fetal academia when FHR accelerations are not seen in the $\mathrm{CTG}^{[3]}$. Of the FSST described, the DST was found to have the highest likely hood ratio for a positive test and the lowest likelihood ratio for a negative test, with Allis clamping of fetal scalp being the second best among the FSST ${ }^{[3]}$

The aim of this study was to determine the effectiveness of the admission CTG combined with FSST during early labour or induction of labour (IOL) to predict or rule out possible underlying fetal hypoxia. This would be helpful in planning further intra partum monitoring. 


\section{METHOD}

The study was carried out at the Academic Obstetrics and Gynaecology unit of the Teaching Hospital Mahamodara, Galle (THMG) from 31 August 2010 to 31 March 2011. Ethical approval was obtained from the Ethical Review Committee of the Faculty of Medicine, University of Ruhuna and the Director of the THMG. Informed written consent was obtained from all the participants $(n=243)$.

Women with singleton uncomplicated pregnancies with a vertex presentation who established spontaneous onset of labour (SOL) defined as painful uterine contractions occurring at intervals of three minutes or less and a cervical os dilated to three centimeters or more and women who had IOL with a cervical os dilated to three centimeters or more, were recruited for the study. Women with pre labour rupture of membranes and those who had or developed fever during labour were excluded. A previous study carried out in the unit had demonstrated that the addition of the FAST to the CTG in the early intra partum period had a specificity of $97 \%$ and a sensitivity of $100 \%$ in predicting an APGAR of $<7$ in neonates at delivery , which was found in $8 \%$ of low risk women ${ }^{[8]}$. Expecting the specificity and sensitivity of the scalp stimulation tests in predicting an APGAR of $<7$ in the neonate at delivery to be approximately $95 \%$ each, and to detect neonatal hypoxia (APGAR $<7$ ) of $6 \%$ or more, with a precision of $5 \%$ with a $95 \%$ confidence level, the minimum sample size was calculated to be 228 using the sensitivity and 275 using the specificity ${ }^{[9]}$ Therefore It was decided to recruit a minimum of 228 and a maximum of 275 subjects for the study.

The CTG was started using a SONICAID $^{\mathrm{TM}}$ CTG recorder and ten minutes later, having checked whether accelerations of FHR had occurred, all of the subjects recruited for the study had amniotomy. Then the DST was carried out under sterile conditions with a finger, in between the uterine contractions, by massaging the fetal scalp gently for 15 seconds. The CTG was continued for another five minutes and the presence or absence of accelerations was documented. If no accelerations were observed, an Allis Clamp was applied to the fetal scalp, under sterile conditions for 15 seconds and the CTG was continued for another five minutes. Neither DST nor Allis clamping was included in the routine management of SOL or IOL in the unit at the time of the study. Therefore, without considering the results of DST or DST and application of an Allis clamp, all the mothers were allowed to progress in labour with either intermittent auscultation of FHR with the Pinnard fetal stethoscope only, or with additional intermittent CTGs as per routine practice, depending on the case. If fetal distress was detected, appropriate management was under taken according to management guidelines in the unit. The mode of delivery and 5 minutes APGAR score of the babies were documented. Five minute APGAR $<7$ was considered to indicate possible underlying fetal hypoxia. The data was stored confidentially in an online computer database and analyzed using SPSS version 17 and Epi Info version 3.4.3.

\section{RESULTS}

The mean age of the study population $(\mathrm{n}=$ 243) was 27.5 yrs ( $95 \% \mathrm{CI}=26.9-28.1)$ with a range of $18-37$ years. Of the 243 subjects $56 \%$ were primigravidae

Out of the 243 patients $224 \quad(92 \%)$ delivered babies with APGAR of $\geq 7$. Of the 107 women who had a no FHR accelerations in the admission CTG, 57 (53\%) had FHR accelerations after the DST (Table1).

Of the 50 women who had no FHR accelerations in the admission CTGs following DST, and therefore had Allis clamping of the fetal scalp, in 27 (54\%), FHR accelerations were observed after the application of the Allis clamp. The 15 women who continued to have no FHR accelerations in the admission CTG, even after DST and Allis clamping of the fetal scalp, delivered babies with APGAR $<7$

Of the 224 women who delivered babies with APGAR $\geq 7$, eight (3.6\%) had no in FHR accelerations in the CTG after FSST. Of the 19 women who delivered babies with APGAR $<7$, four had FHR accelerations in the CTG after FSST. (Table 2)

Of the 35 women who had meconium at amniotomy, 11 underwent subsequent emergency caesarean section (CS) for intra partum fetal distress detected by a subsequent CTG, and two of their neonates had APGAR $<7$ and were admitted to the SCBU for observation. One of them had accelerations following

\section{FSST (Table 3).}

Out of the 19 neonates who had APGAR < 7 , meconium stained liquor was detected in 10 at delivery, two of whom had had meconium at amniotomy while the other eight had de novo meconium. Two of these neonates had had FHR accelerations following FSST (Table 4). No peripartum deaths were observed

The specificity and the PPV of the admission CTG in predicting an APGAR $<7$ increased from $58 \%$ to $96 \%$, and $12 \%$ to $65 \%$ respectively after the addition of the FSST (Table 5)

\section{DISCUSSION}

The absence of FHR accelerations following FSST markedly increased the likelihood of the occurrence of APGAR $<7$ in the neonate and therefore the combination of the CTG with FSST would enable the identification of fetuses who would require close monitoring. Studies done in the United States of America have shown that the DST could have a $100 \%$ sensitivity and negative predictive value in predicting intra partum hypoxia $^{[5,6]}$. In those studies hypoxia was confirmed by fetal scalp $\mathrm{pH}(\mathrm{pH}<7.2)$ which was carried out immediately after the DST. In a previous study conducted in the same unit, the FAST carried in the early intra-partum period demonstrated a sensitivity of $100 \%$ and no false negatives (FHR accelerations) in the fetuses who had a neonatal APGAR $<7^{[8]}$.

The likely hood ratio of a negative DST in the meta-analysis was comparable to the likely hood ratio of a negative DST in our study. Although the likely hood ratio of a positive DST was only $5(95 \%$ CI 3.5 $7.4)$ in our study where as it was 15.68 (95\% CI 3.22-76.24) in the meta-analysis, the combined FSST in our study had a likelihood ratio of a negative test of 0.22 (95\% CI 0.1-0.52) which is comparable with the results of the DST in the metaanalysis and the likelihood ratio of a positive test of 22.1( $95 \%$ CI 10.8-45.4), was better than the results of the DST in the meta- analysis $(15.68,95 \%$ CI 3.22 76.24) [3] (Table 6).

Compared to the APGAR score which is subjective and liable to inter and intra observer variations, the umbilical cord $\mathrm{pH}$ at delivery would have been a more reliable assessment of underlying fetal hypoxia. However facilities for estimation 
of cord blood $\mathrm{pH}$ was not available in the unit during the period of study. It is also important to note that in the current study, four out of the 19 fetuses who had a neonatal APGAR $<7$ demonstrated FHR accelerations following FSST. This is probably due to the fact that the FSST was carried out in early SOL or at IOL, and subsequent intra partum hypoxia can occur due to other reasons including oxytocin infusions and these could lead to a low APGAR at birth. In spite of these limitations, the addition of FSST would not only increase the value of an admission CTG, but could also reduce the duration of the CTG especially in settings with limited resourc

\section{CONCLUSION}

When FHR accelerations are absent in the admission CTG, fetal scalp stimulation tests complement it by increasing its specificity and helping to rule out possible fetal hypoxia during early labour or IOL. Therefore the combination of the CTG with FSST would increase the value of an admission CTG and enable the identification of fetuses who would require close monitoring.

\section{CONFLICTS OF INTEREST}

The authors have no conflicts of interest

\section{REFERENCES}

1. Devane D, Lalor JG, Daly S, McGuire W, Smith V.Cardiotocography versus intermittent auscultation of fetal heart on admission to labour ward for assessment of fetal wellbeing. Cochrane Database of Systematic Reviews 2012, Issue 2. Art. No.: CD005122. DOI: 10.1002/14651858. CD005122.pub4.

2. Wiberg-Itzel E, Lipponer C, Norman $\mathrm{M}$, et al , Determination of $\mathrm{pH}$ or lactate in fetal scalp blood in management of intrapartum fetal distress: randomised controlled multicentre trial.BMJ2008;;336:1284 -1291 .

3. Skupski DW, Rosenberg C, Eglinton GS. Intrapartum fetal Stimulation tests: A meta- analysis. Obstetric and Gynaecology 2002; 99(1) 129134..DOI 10.1016/S0029-7844(01)01645-3.

4. Rathore AM, Ramji S, Bijayalakshmi Devi C, Saini S, Manaktala U, Batra S. Fetal scalp stimulation test: An adjunct tointermittent auscultation in non-reassuring fetal status during labor. The Journal of Obstetrics and Gynaecology Research 2011.DOI: 10.1111/j.1447-0756.2010.01442.

5. Elimain A, Figueroa R ,Tejani N. Intrapartum assessment of fetal well -being: A comparison of scalp stimulation with scalp blood $\mathrm{pH}$ sampling. Obstetric and Gynaecology 1997; 89(3): 373-376 .DOI 10.1016/ S0029-7874(96)-00525

6. Clark SL, Gimovsky ML, Miller FC. The scalp stimulation test: A clinical alternative to fetal scalp blood sampling. American Journal of Obstetrics and Gynecology. 1984; 148: 274-277.

7. Goonewardene Malik, Batcha TM. The fetal acoustic stimulation test : a reliable and cost effective method of antepartum fetal monitoring. Ceylon Medical Journal 2005; 50 (iv): 156159.

8. Goonewardene $\mathrm{M}$, Hanwellage $\mathrm{K}$. Fetal acoustic stimulation test for early intrapartumfetal monitoring. Ceylon Medical Journal 2011; 56 (i): 14-18

9. Lin N.Sample size calculation for sensitivity and specificity studies. www.kck.usm.my/.../samplesize for sensitivity specificity studies. [accessed 29 June 2010]

Table 1 Admission Cardiotocograph with Scalp Digital Stimulation Test and APGAR Score $(\mathbf{n}=\mathbf{2 4 3})$

\begin{tabular}{cccccc}
\hline & \multicolumn{2}{c}{ Admission CTG Before DST } & \multicolumn{2}{c}{ Admission CTG After DST } & Total \\
\cline { 2 - 5 } & No FHR Accelerations & $\begin{array}{c}\text { FHR } \\
\text { Accelerations }\end{array}$ & $\begin{array}{c}\text { No FHR } \\
\text { Accelerations }\end{array}$ & FHR Accelerations & \\
\hline APGAR $\geq 7$ & 94 & 130 & 35 & 189 & 224 \\
APGAR $<7$ & 13 & 06 & 15 & 04 & 19 \\
Total & 107 & 136 & 50 & 193 & 243 \\
\hline
\end{tabular}

$\mathrm{CTG}=$ Cardiotocograph; DST $=$ Digital Stimulation Test; FHR $=$ Fetal Heart Rate

Table 2. Fetal scalp stimulation and APGAR score $(n=243)$

\begin{tabular}{cccc}
\hline & \multicolumn{2}{c}{ Admission CTG after FSST* } & \\
\cline { 2 - 3 } & No FHR Accelerations & FHR Accelerations & Total \\
\hline APGAR $\geq 7$ & 8 & 216 & 224 \\
APGAR $<7$ & 15 & 4 & 19 \\
Total & 23 & 220 & 243 \\
\hline
\end{tabular}

$\mathrm{CTG}=$ Cardiotocograph

FHR $=$ Fetal Heart Rate

*FSST $=$ Fetal scalp stimulation tests included the Digital Stimulation Test (DST) combined with Allis clamping if the DST did not result in FHR accelerations. 
Table 3 Analysis of women with meconium stained liquor at amniotomy $(n=35)$

\begin{tabular}{cccc}
\hline & \multicolumn{2}{c}{ Admission CTG after FSST* } & Total \\
\cline { 2 - 3 } & No FHR Accelerations & FHR Accelerations & 33 \\
\hline APGAR $\geq 7$ & 1 & 32 & 2 \\
APGAR $<7$ & 1 & 1 & 35 \\
Total & 2 & 33 & \\
\hline
\end{tabular}

CTG $=$ Cardiotocograph; FSST $=$ Fetal Scalp Stimulation Test FHR $=$ Fetal Heart Rate

Table 4 Analysis of neonates with APGAR $<7$ at delivery $(n=19)$

\begin{tabular}{cccc}
\hline & \multicolumn{2}{c}{ Admission CTG after FSST* } & Total \\
\cline { 2 - 3 } & No FHR Accelerations & FHR Accelerations & 10 \\
\hline $\begin{array}{c}\text { Meconium present } \\
\text { At delivery } \\
\text { Meconium absent } \\
\text { At delivery } \\
\text { Total }\end{array}$ & 8 & 2 & 9 \\
\hline
\end{tabular}

CTG $=$ Cardiotocograph; FSST $=$ Fetal Scalp Stimulation Test $;$ FHR $=$ Fetal Heart Rate

Table 5. Ability of cardiotocography before and after fetal scalp stimulation tests to predict neonatal asphyxia $($ APGAR < 7) $(n=243)$

\begin{tabular}{|c|c|c|c|}
\hline & $\begin{array}{l}\text { Admission CTG } \\
\qquad(\mathrm{n}=243)\end{array}$ & $\begin{array}{c}\text { Admission CTG after } \\
\text { FSST }^{*} \\
(\mathrm{n}=243)\end{array}$ & $\begin{array}{c}\text { Difference } \\
(95 \% \mathrm{CI}) \\
\mathrm{p}\end{array}$ \\
\hline $\begin{array}{l}\text { Sensitivity } \\
(95 \% \mathrm{CI})\end{array}$ & $\begin{array}{c}68.4 \% \\
(43.5-87.4 \%)\end{array}$ & $\begin{array}{c}79 \% \\
(54.4-94 \%)\end{array}$ & $\begin{array}{c}10.6 \% \\
(2.9-19 \%) \\
0.0061\end{array}$ \\
\hline $\begin{array}{l}\text { Specificity } \\
(95 \% \mathrm{CI})\end{array}$ & $\begin{array}{c}58 \% \\
(51.3-64.6 \%)\end{array}$ & $\begin{array}{c}96.4 \% \\
(93.1-98.5 \%)\end{array}$ & $\begin{array}{c}38.4 \% \\
(30.9-44.8 \%) \\
<0.0001\end{array}$ \\
\hline $\begin{array}{l}\text { Positive Predictive Value } \\
\qquad(95 \% \mathrm{CI})\end{array}$ & $\begin{array}{c}12.2 \% \\
(6.6-19.9 \%)\end{array}$ & $\begin{array}{c}65.2 \% \\
(42.7-83.6 \%)\end{array}$ & $\begin{array}{c}53 \% \\
(45.2-60.1 \%) \\
<0.0001\end{array}$ \\
\hline $\begin{array}{l}\text { Negative Predictive Value } \\
\qquad(95 \% \mathrm{CI})\end{array}$ & $\begin{array}{c}95.6 \% \\
(90.6-98.4 \%)\end{array}$ & $\begin{array}{c}98.1 \% \\
(95.4-99.5 \%)\end{array}$ & $\begin{array}{c}2.5 \% \\
(-0.6-6.8 \%) \\
0.072\end{array}$ \\
\hline $\begin{array}{l}\text { Likelihood Ratio of a } \\
\text { Positive Test }(95 \% \text { CI) }\end{array}$ & $\begin{array}{c}1.6 \\
(1.2-2.3)\end{array}$ & $\begin{array}{c}22.1 \\
(10.8-45.4)\end{array}$ & \\
\hline $\begin{array}{l}\text { Likelihood Ratio of a } \\
\text { Negative Test }(95 \% \text { CI) }\end{array}$ & $\begin{array}{c}0.54 \\
(0.3-1.1)\end{array}$ & $\begin{array}{c}0.22 \\
(0.1-0.52)\end{array}$ & \\
\hline Prevalence $(95 \% \mathrm{CI})$ & $\begin{array}{c}7.8 \\
(4.8-11.9)\end{array}$ & $\begin{array}{c}7.8 \\
(4.8-11.9)\end{array}$ & \\
\hline
\end{tabular}

$\mathrm{CTG}=$ Cardiotocograph

DST $=$ Digital Stimulation Test

*FSST $=$ Fetal scalp stimulation tests included DST combined with Allis clamping if DST did not result in fetal heart rate accelerations: $95 \% \mathrm{CI}=95 \%$ Confidence Interval 
Table 6.Likelyhood Ratios of Fetal scalp stimulation tests

Likelihood Ratio of a positive test $(95 \%$

CI)

Allis clamp[3]

$\operatorname{DST}[3]$

DST current study

Allis clamp +DST current study

DST $=$ Digital Stimulation Test
$10.4(1.47-73.61)$

$15.68(3.22-76.24)$

$5(3.5-7.4)$

$22.1(10.8-45.4)$

Likelihood Ratio of a negative test $(95 \% \mathrm{CI})$

$0.10(0.01-0.68)$

$0.06(0.01-0.31)$

$0.25(0.1-0.6)$

$0.22(0.1-0.52)$ 